\title{
Fibonacci Collocation Method for Solving High-Order Linear Fredholm Integro-Differential-Difference Equations
}

\author{
Ayşe Kurt, Salih Yalçınbaş, and Mehmet Sezer \\ Department of Mathematics, Celal Bayar University, Muradiye, 45140 Manisa, Turkey \\ Correspondence should be addressed to Salih Yalçınbaş; salih.yalcinbas@cbu.edu.tr
}

Received 8 May 2013; Accepted 27 June 2013

Academic Editor: Irena Lasiecka

Copyright (C) 2013 Ayşe Kurt et al. This is an open access article distributed under the Creative Commons Attribution License, which permits unrestricted use, distribution, and reproduction in any medium, provided the original work is properly cited.

A new collocation method based on the Fibonacci polynomials is introduced for the approximate solution of high order-linear Fredholm integro-differential-difference equations with the mixed conditions. The proposed method is analyzed to show the convergence of the method. Some further numerical experiments are carried out to demonstrate the method.

\section{Introduction}

The integro-differential-difference equations (IDDEs) have been developed very rapidly in recent years. This is an important branch of mathematics which has a lot of interest in many application fields such as engineering, mechanics, physics, astronomy, chemistry, biology, economics, and potential theory, electrostatics [1-14]. Since some IDDEs are hard to solve numerically, they are solved by using the approximated methods. Several numerical methods were used such as the successive approximations, Adomian decomposition, Haar Wavelet, and Tau and Walsh series methods [15-20]. Additionally the Monte Carlo method for linear Fredholm integro-differential-difference equation has been presented by Farnoosh and Ebrahimi [21] and the Direct method based on the Fourier and block-pulse method functions by Asady et al. [22].

Since the beginning of 1994, the Taylor and Chebyshev matrix methods have also been used by Sezer et al. to solve linear differential, Fredholm integral, and Fredholm integro-differential equations [23-35]. Lately, the Fibonacci collocation method has been used to find the approximate solutions of differential, integral, and integro-differential equations [36].
In this study, we consider the approximate solution of the $m$ th-order Fredholm integro-differential-difference equations,

$$
\begin{gathered}
\sum_{k=0}^{m} P_{k}(x) y^{(k)}(x)+\sum_{r=0}^{s} Q_{r}(x) y^{(r)}\left(\mu_{r} x+\tau_{r}\right) \\
=\mathrm{g}(x)+\lambda \int_{a}^{b} K(x, t) y(t) d t,
\end{gathered}
$$

where $s \leq m, \tau_{r}$ are the integer, $0 \leq a \leq x, t \leq b$, under the mixed conditions

$$
\sum_{k=0}^{m-1}\left[a_{j k} y^{(k)}(a)+b_{j k} y^{(k)}(b)\right]=\lambda_{j}, \quad j=1,2,3, \ldots, m,
$$

where $P_{k}(x), Q_{r}(x), g(x)$, and $K(x, t)$ are functions defined on $a \leq x, t \leq b ; a_{j k}, b_{j k}, \lambda$, and $\lambda_{j}$ are suitable constants.

Our aim is to obtain an approximate solution of (1) expressed in the truncated Fibonacci series form:

$$
y(x)=\sum_{n=1}^{N} a_{n} F_{n}(x),
$$


where $a_{n}, n=1,2,3, \ldots, N$, are the unknown Fibonacci coefficients. Here $N$ is positive integer such that $N \geq m$ and $F_{n}(x), n=1,2,3, \ldots, N$, are the Fibonacci polynomials defined by

$$
\begin{aligned}
& F_{n}(x)=\sum_{j=0}^{[(n-1) / 2]}\left(\begin{array}{c}
n-j-1 \\
j
\end{array}\right) x^{n-2 j-1}, \\
& {\left[\frac{(n-1)}{2}\right]= \begin{cases}\frac{(n-2)}{2}, & n \text { even }, \\
\frac{(n-1)}{2}, & n \text { odd } .\end{cases} }
\end{aligned}
$$

\section{Fundamental Matrix Relations}

Firstly, we can write the Fibonacci polynomials $F_{n}(x)$ in the matrix form as follows:

$$
\mathbf{F}^{T}(x)=\mathbf{C X}^{T}(x) \Longleftrightarrow \mathbf{F}(x)=\mathbf{X}(x) \mathbf{C}^{T},
$$

where

$$
\begin{gathered}
\mathbf{F}(x)=\left[\begin{array}{llll}
F_{1}(x) & F_{2}(x) & \cdots & F_{N}(x)
\end{array}\right], \\
\mathbf{X}(x)=\left[\begin{array}{lll}
1 x & \cdots & x^{N-1}
\end{array}\right] .
\end{gathered}
$$

If $N$ is even,

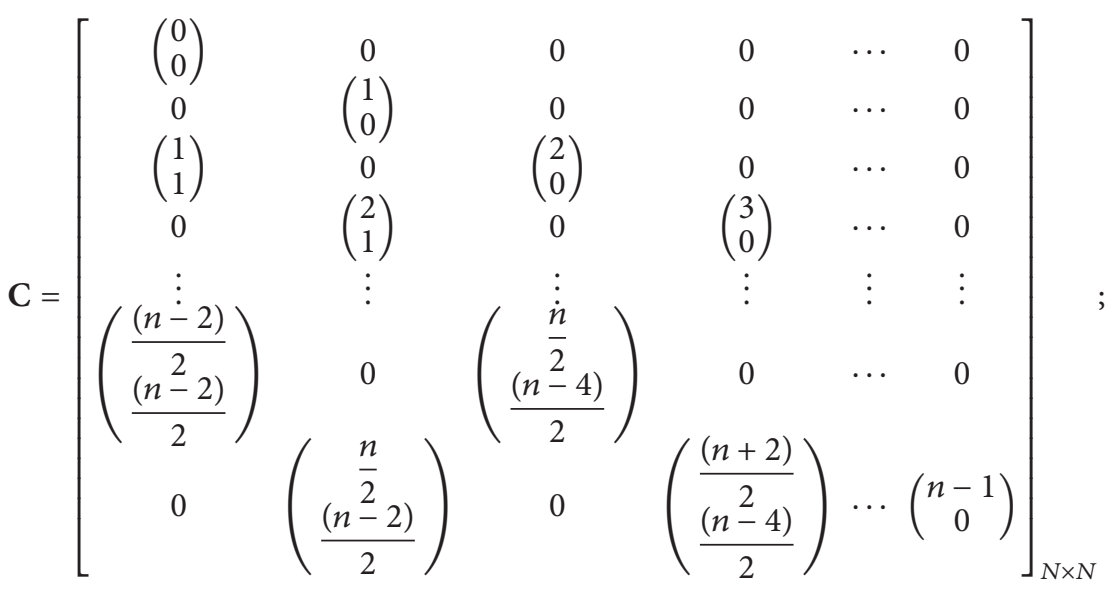

if $N$ is odd,

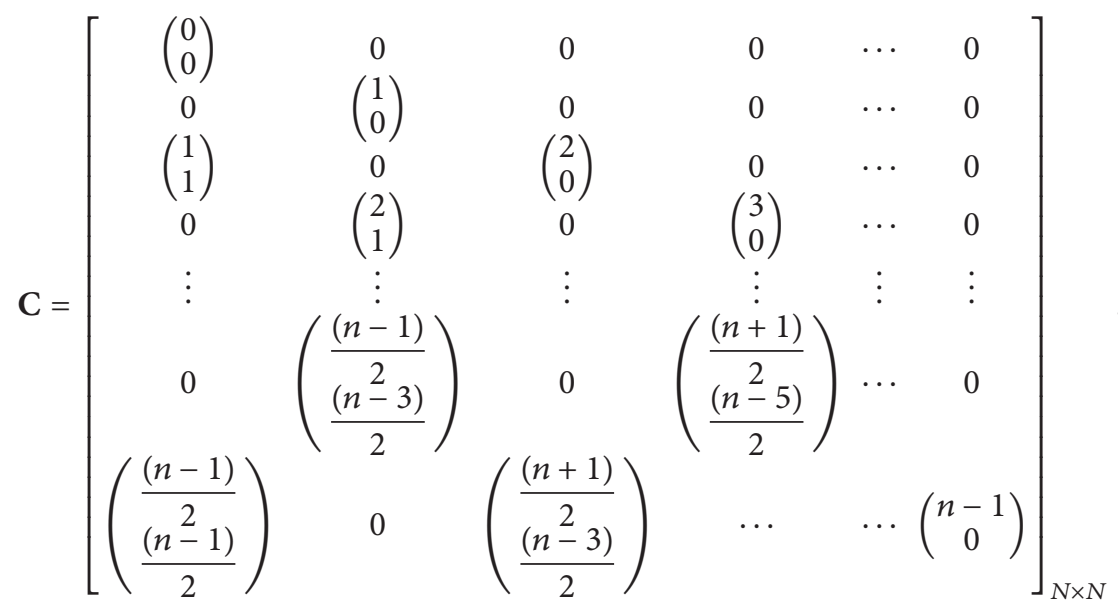

Let us show (1) in the following form:

$$
P(x)+Q(x)=\mathrm{g}(x)+\lambda I(x),
$$

where

$$
P(x)=\sum_{k=0}^{m} P_{k}(x) y^{(k)}(x),
$$

$$
Q(x)=\sum_{r=0}^{s} Q_{r}(x) y^{(r)}\left(\mu_{r} x+\tau_{r}\right)
$$

$$
I(x)=\lambda \int_{a}^{b} K(x, t) y(t) d t
$$


2.1. Matrix Relations for the Differential Part $P(x)$. Firstly, we consider the solution $\mathbf{y}(x)$ and its $k$ th derivate $\mathbf{y}^{(k)}(x)$ in the matrix form:

$$
\begin{gathered}
\mathbf{y}(x)=\mathbf{F}(x) \mathbf{A}, \quad \mathbf{A}=\left[\begin{array}{llll}
a_{1} & a_{2} & \cdots & a_{N}
\end{array}\right]^{T}, \\
\mathbf{y}^{(k)}(x)=\mathbf{F}^{(k)}(x) \mathbf{A} .
\end{gathered}
$$

Then, from relations (5) and (11), we can obtain the following matrix form:

$$
\mathbf{y}(x)=\mathbf{X}(x) \mathbf{C}^{T} \mathbf{A} .
$$

Similar to (13), from relations (5), (11), and (12), we can find $\mathbf{y}^{(k)}(x)$ matrix form as

$$
\mathbf{y}^{(k)}(x)=\mathbf{X}^{(k)}(x) \mathbf{C}^{T} \mathbf{A} .
$$

To find the matrix $\mathbf{X}^{(k)}(x)$ in terms of the matrix $\mathbf{X}(x)$, we can use the following relation:

$$
\begin{gathered}
\mathbf{X}^{(1)}(x)=\mathbf{X}(x) \mathbf{T}^{T}, \\
\mathbf{X}^{(2)}(x)=\mathbf{X}^{(1)}(x) \mathbf{T}^{T}=\left(\mathbf{X}(x) \mathbf{T}^{T}\right) \mathbf{T}^{T}=\mathbf{X}(x)\left(\mathbf{T}^{T}\right)^{2} \\
\vdots \\
\mathbf{X}^{(k)}(x)=\mathbf{X}^{(k-1)}(x) \mathbf{T}^{T}=\mathbf{X}(x)\left(\mathbf{T}^{T}\right)^{k},
\end{gathered}
$$

where

$$
\mathbf{T}^{T}=\left[\begin{array}{ccccccc}
0 & 1 & 0 & \cdots & 0 & 0 & 0 \\
0 & 0 & 2 & \cdots & 0 & 0 & 0 \\
0 & 0 & 0 & \cdots & 0 & 0 & 0 \\
\vdots & \vdots & \vdots & & \vdots & & \vdots \\
0 & 0 & 0 & \cdots & 0 & 0 & N-1 \\
0 & 0 & 0 & \cdots & 0 & 0 & 0
\end{array}\right] .
$$

Subsequently, by substituting the matrix form (15) into (14), we obtain the matrix relations

$$
\mathbf{y}^{(k)}(x)=\mathbf{X}(x)\left(\mathbf{T}^{T}\right)^{k} \mathbf{C}^{T} \mathbf{A} .
$$

2.2. Matrix Relations for the Difference Part $Q(x)$. If we put $x \rightarrow \mu_{r} x+\tau_{r}$ in the relation (11), we have the matrix form

$$
\mathbf{y}\left(\mu_{r}+\tau_{r}\right)=\mathbf{F}\left(\mu_{r} x+\tau_{r}\right) \mathbf{A} .
$$

It is seen that the relation between the matrices $\mathbf{X}(x)$ and $\mathbf{X}\left(\mu_{r} x+\tau_{r}\right)$ is

$$
\mathbf{X}\left(\mu_{r} x+\tau_{r}\right)=\mathbf{X}(x) \boldsymbol{\beta}\left(\mu_{r}, \tau_{r}\right),
$$

where

$$
\begin{aligned}
& \boldsymbol{\beta}\left(\mu_{r}, \tau_{r}\right) \\
& =\left[\begin{array}{cccc}
\left(\begin{array}{l}
0 \\
0
\end{array}\right) \mu_{r}{ }^{0} \tau_{r}{ }^{0} & \left(\begin{array}{l}
1 \\
0
\end{array}\right) \mu_{r}{ }^{0} \tau_{r}{ }^{1} & \ldots & \left(\begin{array}{c}
N-1 \\
0
\end{array}\right) \mu_{r}{ }^{0} \tau_{r}{ }^{N-1} \\
0 & \left(\begin{array}{l}
1 \\
1
\end{array}\right) \mu_{r}{ }^{1} \tau_{r}{ }^{0} & \cdots & \left(\begin{array}{c}
N-1 \\
1
\end{array}\right) \mu_{r}{ }^{1} \tau_{r}{ }^{N-2} \\
\vdots & \vdots & \ddots & \vdots \\
0 & 0 & \cdots & \left(\begin{array}{c}
N-1 \\
N-1
\end{array}\right) \mu_{r}{ }^{N-1} \tau_{r}{ }^{0}
\end{array}\right] .
\end{aligned}
$$

By using the relations (15) and (19), we can get

$$
\mathbf{X}^{(k)}\left(\mu_{r} x+\tau_{r}\right)=\mathbf{X}(x) \boldsymbol{\beta}\left(\mu_{r}, \tau_{r}\right)\left(\mathbf{T}^{T}\right)^{k} .
$$

Thus from (14) and (21), we can find

$$
\mathbf{y}^{(k)}\left(\mu_{r}+\tau_{r}\right)=\mathbf{X}(x) \boldsymbol{\beta}\left(\mu_{r}, \tau_{r}\right)\left(\mathbf{T}^{T}\right)^{k} \mathbf{C}^{T} \mathbf{A} .
$$

By using the expressions (14) and (22), we obtain the matrix form

$$
\begin{gathered}
P(x)=\sum_{k=0}^{m} p_{k}(x) \mathbf{X}(x)\left(\mathbf{T}^{T}\right)^{k} \mathbf{C}^{T} \mathbf{A}, \\
Q(x)=\sum_{r=0}^{s} Q_{r}(x) \mathbf{X}(x) \boldsymbol{\beta}\left(\mu_{r}, \tau_{r}\right)\left(\mathbf{T}^{T}\right)^{r} \mathbf{C}^{T} \mathbf{A} .
\end{gathered}
$$

2.3. Matrix Relations for the Integral Part. Let us find the matrix relation for the Fredholm integral part $I(x)$ in (9). The kernel function $K(x, t)$ can be shown by the truncated Fibonacci series,

$$
K(x, t)=\sum_{m=0}^{N} \sum_{n=0}^{N} k_{m n}^{f} F_{m}(x) F_{n}(t),
$$

and the truncated Taylor series,

$$
K(x, t)=\sum_{m=0}^{N} \sum_{n=0}^{N} k_{m n}^{t} x^{m} t^{n},
$$

where

$$
k_{m n}^{t}=\frac{1}{m ! n !} \frac{\partial^{m+n} K(0,0)}{\partial x^{m} \partial x^{n}} ; \quad m, n=0,1, \ldots, N .
$$

The expressions (24) and (25) can be put in matrix forms as

$$
\begin{array}{r}
K(x, t)=\mathbf{F}(x) \mathbf{K}_{F} \mathbf{F}^{T}(t), \quad \mathbf{K}_{F}=\left[k_{m n}^{F}\right], \\
m, n=0,1, \ldots, N, \\
K(x, t)=\mathbf{X}(x) \mathbf{K}_{t} \mathbf{X}^{T}(t), \quad \mathbf{K}_{t}=\left[k_{m n}^{t}\right], \\
m, n=0,1, \ldots, N .
\end{array}
$$


From (11), (27), and (28) we can obtain

$$
\begin{aligned}
\mathbf{X}(x) \mathbf{K}_{t} \mathbf{X}^{T}(t) & =\mathbf{F}(x) \mathbf{K}_{F} \mathbf{F}^{T}(t) \\
& \Longrightarrow \mathbf{X}(x) \mathbf{K}_{t} \mathbf{X}^{T}(t)=\mathbf{X}(x) \mathbf{C}^{T} \mathbf{K}_{F} \mathbf{C} \mathbf{X}^{T}(t) .
\end{aligned}
$$

Thus

$$
\mathbf{K}_{t}=\mathbf{C}^{T} \mathbf{K}_{F} \mathbf{C} \Longrightarrow \mathbf{K}_{F}=\left(\mathbf{C}^{T}\right)^{-1} \mathbf{K}_{t} \mathbf{C}^{-1}
$$

By substituting the matrix forms (22) and (27) into the integral part $I(x)$ in (9), we can have the matrix relation as follows:

$$
\begin{aligned}
{[I(x)] } & =\int_{a}^{b} \mathbf{F}(x) \mathbf{K}_{F} \mathbf{F}^{T}(t) \mathbf{X}(t) \boldsymbol{\beta}\left(\mu_{r}, \tau_{r}\right)\left(\mathbf{T}^{T}\right)^{k} \mathbf{C}^{T} \mathbf{A} d t \\
& =\mathbf{F}(x) \mathbf{K}_{F} \mathbf{Q A}
\end{aligned}
$$

so that

$$
\mathbf{Q}=\int_{a}^{b} \mathbf{F}^{T}(t) \mathbf{X}(t) \boldsymbol{\beta}\left(\mu_{r}, \tau_{r}\right)\left(\mathbf{T}^{T}\right)^{k} \mathbf{C}^{T} d t
$$

From (5) and (32), we have

$$
\begin{aligned}
\mathbf{Q} & =\int_{a}^{b} \mathbf{C} \mathbf{X}^{T}(t) \mathbf{X}(t) \boldsymbol{\beta}\left(\mu_{r}, \tau_{r}\right)\left(\mathbf{T}^{T}\right)^{k} \mathbf{C}^{T} d t \\
& =\mathbf{C H} \boldsymbol{\beta}\left(\mu_{r}, \tau_{r}\right)\left(\mathbf{T}^{T}\right)^{k} \mathbf{C}^{T},
\end{aligned}
$$

where

$$
\begin{gathered}
\mathbf{H}=\int_{a}^{b} \mathbf{X}^{T}(t) \mathbf{X}(t) d t, \quad \mathbf{H}=\left[h_{i j}\right], \\
h_{i j}=\frac{b^{i+j+1}-a^{i+j+1}}{i+j+1} i, \quad j=1,2, \ldots, N .
\end{gathered}
$$

If we substitute the matrix relation (5) into (31), we have the matrix form

$$
[\mathbf{I}(x)]=\mathbf{X}(x) \mathbf{C}^{T} \mathbf{K}_{F} \mathbf{Q A} .
$$

2.4. Matrix Relations for the Conditions. The corresponding matrix form for the conditions (2) can be shown, by means of (17), as

$$
\sum_{k=0}^{m-1}\left[a_{j k} \mathbf{X}(a)+b_{j k} \mathbf{X}(b)\right]\left(\mathbf{T}^{T}\right)^{k} \mathbf{C}^{T} \mathbf{A}=\lambda_{j}, \quad j=1,2, \ldots, m .
$$

\section{Method of Solution}

We can construct the fundamental matrix equation corresponding for (1). For this aim, we substitute the matrix relations (23) and (35) into (9). So we obtain the matrix equation

$$
\begin{aligned}
\sum_{k=0}^{m} P_{k}(x) & \mathbf{X}(x) \mathbf{C}^{T}\left(\mathbf{T}^{T}\right)^{k} \mathbf{A} \\
& +\sum_{r=0}^{s} Q_{r}(x) \mathbf{X}(x) \boldsymbol{\beta}\left(\mu_{r}, \tau_{r}\right)\left(\mathbf{T}^{T}\right)^{r} \mathbf{C}^{T} \mathbf{A} \\
= & g(x)+\lambda \mathbf{X}(x) \mathbf{C}^{T} \mathbf{K}_{F} \mathbf{Q A} .
\end{aligned}
$$

By using in (37) the collocation points $x_{i}$ defined by

$$
x_{i}=a+\left(\frac{b-a}{N-1}\right)(i-1), \quad i=1,2, \ldots, N
$$

the system of the matrix equations is obtained

$$
\begin{aligned}
\sum_{k=0}^{m} P_{k}\left(x_{i}\right) & \mathbf{X}\left(x_{i}\right) \mathbf{C}^{T}\left(\mathbf{T}^{T}\right)^{k} \mathbf{A} \\
& +\sum_{r=0}^{s} Q_{r}\left(x_{i}\right) \mathbf{X}\left(x_{i}\right) \boldsymbol{\beta}\left(\mu_{r}, \tau_{r}\right)\left(\mathbf{T}^{T}\right)^{r} \mathbf{C}^{T} \mathbf{A} \\
= & \mathrm{g}\left(x_{i}\right)+\lambda \mathbf{X}\left(x_{i}\right) \mathbf{C}^{T} \mathbf{K}_{F} \mathbf{Q A}
\end{aligned}
$$

or shortly the fundamental matrix equation becomes

$$
\begin{aligned}
& \left\{\sum_{k=0}^{m} \mathbf{P}_{k} \mathbf{X}\left(\mathbf{T}^{T}\right)^{k} \mathbf{C}^{T}+\sum_{r=0}^{s} \mathbf{Q}_{r} \mathbf{X} \boldsymbol{\beta}\left(\mu_{r}, \tau_{r}\right)\left(\mathbf{T}^{T}\right)^{r} \mathbf{C}^{T}\right. \\
& \left.-\lambda \mathbf{X} \mathbf{C}^{T} \mathbf{K}_{F} \mathbf{Q}\right\} \mathbf{A}=\mathbf{G},
\end{aligned}
$$

where

$$
\begin{gathered}
\mathbf{P}_{k}=\left[\begin{array}{cccc}
p_{k}\left(x_{1}\right) & 0 & \cdots & 0 \\
0 & p_{k}\left(x_{2}\right) & \cdots & 0 \\
0 & 0 & \ddots & 0 \\
0 & 0 & \cdots & p_{k}\left(x_{N}\right)
\end{array}\right], \\
\mathbf{Q}_{r}=\left[\begin{array}{cccc}
Q_{r}\left(x_{1}\right) & 0 & \cdots & 0 \\
0 & Q_{r}\left(x_{2}\right) & \cdots & 0 \\
0 & 0 & \ddots & 0 \\
0 & 0 & \cdots & Q_{r}\left(x_{N}\right)
\end{array}\right], \\
\mathbf{X}=\left[\begin{array}{c}
X\left(x_{1}\right) \\
X\left(x_{2}\right) \\
\vdots \\
X\left(x_{N}\right)
\end{array}\right]=\left[\begin{array}{cccc}
1 & x_{1} & \cdots & x_{1}^{N-1} \\
1 & x_{2} & \cdots & x_{2}^{N-1} \\
\vdots & \vdots & \ddots & \vdots \\
1 & x_{N} & \cdots & x_{N}^{N-1}
\end{array}\right], \\
\mathbf{G}=\left[\begin{array}{c}
\mathrm{g}\left(x_{1}\right) \\
\mathrm{g}\left(x_{2}\right) \\
\vdots \\
\mathrm{g}\left(x_{N}\right)
\end{array}\right] .
\end{gathered}
$$

Therefore, the fundamental matrix equation (40) corresponding for (1) can be written as

$$
\mathbf{W A}=\mathbf{G} \quad \text { or } \quad[\mathbf{W} ; \mathbf{G}],
$$


where

$$
\begin{aligned}
\mathbf{W}= & \sum_{k=0}^{m} \mathbf{P}_{k} \mathbf{X}\left(\mathbf{T}^{T}\right)^{k} \mathbf{C}^{T} \\
& +\sum_{r=0}^{s} \mathbf{Q}_{r} \mathbf{X} \boldsymbol{\beta}\left(\mu_{r}, \tau_{r}\right)\left(\mathbf{T}^{T}\right)^{r} \mathbf{C}^{T}-\lambda \mathbf{X} \mathbf{C}^{T} \mathbf{K}_{F} \mathbf{Q} .
\end{aligned}
$$

Equation (42) corresponds to a system of $N$ linear algebraic equations with unknown Fibonacci coefficients $a_{1}, a_{2}, \ldots, a_{N}$. Further, we can express the matrix form (36) conditions

$$
\mathbf{U}_{j} A=\left[\lambda_{j}\right] \quad \text { or } \quad\left[\mathbf{U}_{j} ; \lambda_{j}\right], \quad j=1,2, \ldots, m,
$$

where

$$
\begin{aligned}
\mathbf{U}_{j} & =\sum_{k=0}^{m-1}\left[a_{j k} \mathbf{X}(a)+b_{j k} \mathbf{X}(b)\right]\left(\mathbf{T}^{T}\right)^{k} \mathbf{C}^{T} \\
& =\left[\begin{array}{lllll}
u_{j 1} & u_{j 2} & u_{j 3} & \ldots & u_{j N}
\end{array}\right] .
\end{aligned}
$$

To obtain the solution of (1) under the conditions (2), by replacing the row matrices (44) by the last $m$ rows of the matrices (42), we have the new augmented matrix

$$
\widetilde{\mathbf{W}} \mathbf{A}=\widetilde{\mathbf{G}} \text {. }
$$

If the last $\mathrm{m}$ rows of the (30) are replaced, the augmented matrix of the above system is obtained as

$[\widetilde{\mathbf{W}} ; \widetilde{\mathbf{G}}]$

$$
=\left[\begin{array}{cccccc}
w_{11} & w_{12} & \cdots & w_{1 N} & ; & \mathrm{g}\left(t_{1}\right) \\
w_{21} & w_{22} & \cdots & w_{2 N} & ; & \mathrm{g}\left(t_{2}\right) \\
\vdots & \vdots & \vdots & \vdots & ; & \vdots \\
w_{(N-m) 1} & w_{(N-m) 2} & \cdots & w_{(N-m) N} & ; & \mathrm{g}\left(t_{N-m}\right) \\
u_{11} & u_{12} & \cdots & u_{1 N} & ; & \lambda_{1} \\
u_{21} & u_{22} & \cdots & u_{2 N} & ; & \lambda_{2} \\
\vdots & \vdots & \vdots & \vdots & ; & \vdots \\
u_{m 1} & u_{m 2} & \cdots & u_{m N} & ; & \lambda_{m}
\end{array}\right] .
$$

If $\operatorname{rank} \widetilde{\mathbf{W}}=\operatorname{rank}[\widetilde{\mathbf{W}} ; \widetilde{\mathbf{G}}]=N$, then we can write

$$
\mathbf{A}=(\widetilde{\mathbf{W}})^{-\mathbf{1}} \widetilde{\mathbf{G}} \text {. }
$$

And so, the matrix $\mathbf{A}$ (thereby the coefficients $a_{1}, a_{2}, \ldots, a_{N}$ ) is uniquely determined.

\section{Accuracy of Solution}

We can check the accuracy of the method. The truncated Fibonacci series in (3) have to be approximately satisfying (1). For each $x=x_{i} \in[a, b], i=1,2,3, \ldots$,

$$
E\left(x_{i}\right)=\left|P\left(x_{i}\right)-Q\left(x_{i}\right)-\mathrm{g}\left(x_{i}\right)-\lambda I\left(x_{i}\right)\right| \cong 0
$$

or

$$
E\left(x_{i}\right) \leq 10^{-k_{i}} \quad\left(k_{i} \text { is any positive integer }\right) .
$$

If $\max \left(10^{-k_{i}}\right)=10^{-k}$ ( $k$ is any positive integer) is prescribed, then the truncation limit $N$ is increased until the difference $E\left(x_{i}\right)$ at each of the points $x_{i}$ becomes smaller than the prescribed $10^{-k}$.

\section{Numerical Examples}

In this section, several examples are given to illustrate the applicability of the method and all of them are performed on the computer MATLAB. Also, the absolute errors in tables are the values of $\left|y(x)-y_{N}(x)\right|$ at selected points.

Example 1. Let us consider the linear Fredholm integrodifferential-difference equation given by

$$
\begin{aligned}
& y^{\prime \prime}(x)-y^{\prime \prime}(x-1)+2 x y^{\prime}(x-2) \\
& =4 x^{2}-15 x+4+12 \int_{0}^{1} x t y(t) d t, \quad 0 \leq x, t \leq 1,
\end{aligned}
$$

with the boundary conditions

$$
y(0)=1, \quad y(1)=1
$$

and the approximate solution $y(x)$ by the truncated Fibonacci series

$$
y(x)=\sum_{n=1}^{3} a_{n} F_{n}(x),
$$

where $N=3, P_{2}(x)=1, Q_{1}(x)=2 x, Q_{2}(x)=1, \mu_{1}=1$, $\tau_{1}=-2, \mu_{2}=1$, and $\tau_{2}=-1$,

$$
\lambda=12, \quad K(x, t)=x t, \quad g(x)=4 x^{2}-15 x+4 .
$$

From (38), the collocation points for $N=3$, are computed

$$
\left\{x_{1}=0, x_{2}=\frac{1}{2}, x_{3}=1\right\}
$$

and from (40), the fundamental matrix equation of the problem is

$$
\begin{aligned}
& \left\{\mathbf{P}_{2} \mathbf{X}\left(\mathbf{T}^{T}\right)^{2} \mathbf{C}^{T}+\mathbf{Q}_{0} \mathbf{X} \boldsymbol{\beta}(1,-2)\left(\mathbf{T}^{T}\right)^{0} \mathbf{C}^{T}\right. \\
& \left.+\mathbf{Q}_{1} \mathbf{X} \boldsymbol{\beta}(1,-1)\left(\mathbf{T}^{T}\right)^{2} \mathbf{C}^{T}-\lambda \mathbf{X} \mathbf{C}^{T} \mathbf{K}_{f} \mathbf{Q}\right\} \mathbf{A}=\mathbf{G},
\end{aligned}
$$

where

$$
\begin{gathered}
\mathbf{P}_{2}=\left[\begin{array}{lll}
1 & 0 & 0 \\
0 & 1 & 0 \\
0 & 0 & 1
\end{array}\right], \quad \mathbf{Q}_{1}=\left[\begin{array}{lll}
0 & 0 & 0 \\
0 & 1 & 0 \\
0 & 0 & 2
\end{array}\right], \quad \mathbf{Q}_{2}=\left[\begin{array}{lll}
1 & 0 & 0 \\
0 & 1 & 0 \\
0 & 0 & 1
\end{array}\right], \\
\mathbf{T}^{T}=\left[\begin{array}{lll}
0 & 1 & 0 \\
0 & 0 & 2 \\
0 & 0 & 0
\end{array}\right], \quad \mathbf{C}^{T}=\left[\begin{array}{lll}
1 & 0 & 1 \\
0 & 1 & 0 \\
0 & 0 & 1
\end{array}\right], \quad \mathbf{G}=\left[\begin{array}{c}
4 \\
5 \\
-\frac{5}{2} \\
-7
\end{array}\right], \\
\boldsymbol{\beta}(1,-2)=\left[\begin{array}{ccc}
1 & -2 & 4 \\
0 & 1 & -4 \\
0 & 0 & 1
\end{array}\right], \quad \boldsymbol{\beta}(1,-1)=\left[\begin{array}{ccc}
1 & -1 & 1 \\
0 & 1 & -2 \\
0 & 0 & 0
\end{array}\right], \\
\mathbf{K}=\left[\begin{array}{lll}
0 & 0 & 0 \\
0 & 1 & 0 \\
0 & 0 & 0
\end{array}\right], \quad \mathbf{Q}=\left[\begin{array}{ccc}
1 & \frac{1}{2} & \frac{4}{3} \\
\frac{1}{2} & \frac{1}{3} & \frac{3}{4} \\
\frac{4}{\frac{4}{3}} & \frac{3}{4} & \frac{28}{15}
\end{array}\right] .
\end{gathered}
$$


The augmented matrix for this fundamental matrix equation is calculated as

$$
[\mathbf{W} ; \mathbf{G}]=\left[\begin{array}{ccccc}
0 & 0 & 4 & ; & 4 \\
-3 & -1 & -\frac{7}{2} & ; & -\frac{5}{2} \\
-6 & -2 & -9 & ; & -7
\end{array}\right] .
$$

From (37), the matrix forms for the boundary conditions are

$$
\begin{aligned}
& {\left[\mathbf{U}_{0} ; \lambda_{0}\right]=\left[\begin{array}{lllll}
1 & 0 & 1 & ; & 1
\end{array}\right],} \\
& {\left[\mathbf{U}_{1} ; \lambda_{1}\right]=\left[\begin{array}{lllll}
0 & 1 & 2 & ; & 1
\end{array}\right] .}
\end{aligned}
$$

The new augmented matrix based on conditions can be written as

$$
[\widetilde{\mathbf{W}} ; \widetilde{\mathbf{G}}]=\left[\begin{array}{lllll}
0 & 0 & 4 & ; & 4 \\
1 & 0 & 1 & ; & 1 \\
0 & 1 & 2 & ; & 1
\end{array}\right] \text {. }
$$

Solving this system, the unknown Fibonacci coefficients are obtained as

$$
A=\left[\begin{array}{lll}
0 & -1 & 1
\end{array}\right]^{T} \text {. }
$$

Hence, by substituting the Fibonacci coefficients matrix into (11), we have the approximate solution $y(x)=x^{2}-x+1$, which is the exact solution

$$
\begin{aligned}
y(x) & =\sum_{n=1}^{3} a_{n} F_{n}(x)=a_{1} F_{1}(x)+a_{2} F_{2}(x)+a_{3} F_{3}(x) \\
& =0.1+(-1) \cdot x+1 \cdot\left(x^{2}+1\right)=x^{2}-x+1 .
\end{aligned}
$$

Example 2 (see [26]). Consider the following linear Fredholm integro-differential-difference equation with variable coefficients

$$
\begin{aligned}
y^{\prime \prime \prime}(x)- & x y^{\prime}(x)+y^{\prime \prime}(x-1)-x y(x-1) \\
= & -(x+1)(\sin (x-1)+\cos (x)) \\
& \quad-\cos (2)+1+\int_{-1}^{1} y(t-1) d t \quad-1 \leq x, t \leq 1
\end{aligned}
$$

with the conditions $y(0)=0, y^{\prime}(0)=1$, and $y^{\prime \prime}(0)=0$ and the exact solution $y(x)=\sin (x)$.

So $P_{1}(x)=-x, P_{3}(x)=1, Q_{1}(x)=2 x, Q_{2}(x)=1$, $\mu_{1}=1, \tau_{1}=-1, \mu_{0}=1$, and $\tau_{0}=-1$,

$$
\begin{gathered}
\lambda=1, \quad K(x, t)=t-1, \\
\mathrm{~g}(x)=-(x+1)(\sin (x-1)+\cos (x))-\cos (2)+1 .
\end{gathered}
$$

The fundamental matrix equation of the problem from (40) becomes

$$
\begin{aligned}
& \left\{\mathbf{P}_{1} \mathbf{X}\left(\mathbf{T}^{T}\right)^{1} \mathbf{C}^{T}+\mathbf{P}_{3} \mathbf{X}\left(\mathbf{T}^{T}\right)^{3} \mathbf{C}^{T}+\mathbf{Q}_{0} \mathbf{X} \boldsymbol{\beta}(1,-1)\left(\mathbf{T}^{T}\right)^{0} \mathbf{C}^{T}\right. \\
& \left.+\mathbf{Q}_{1} \mathbf{X} \boldsymbol{\beta}(1,-1)\left(\mathbf{T}^{T}\right)^{2} \mathbf{C}^{T}-\lambda \mathbf{X} \mathbf{C}^{T} \mathbf{K}_{f} \mathbf{Q}\right\} \mathbf{A}=\mathbf{G} .
\end{aligned}
$$

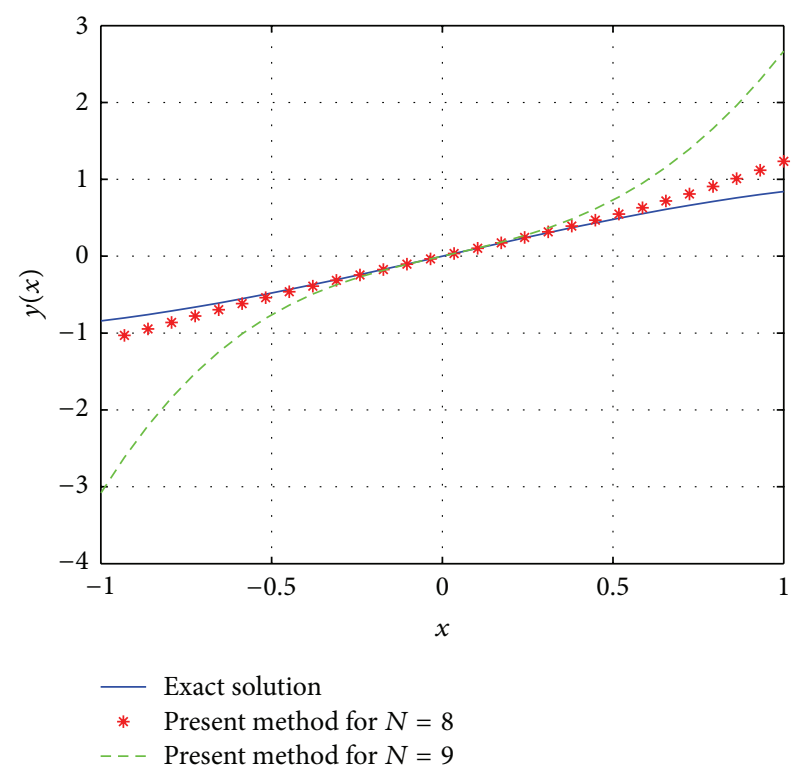

FIGURE 1: Numerical and exact solutions of Example 2 for $N=8,9$.

For $N=8,9$ the solutions of the problem are found as, respectively,

$$
\begin{aligned}
y(x)= & (0.003823057225) x^{7}+(0.01008064682) x^{6} \\
& -(0.02660425406) x^{5}+(0.05046207175) x^{4} \\
& +(0.1974487774) x^{3}-(2.925448512) x^{2} \\
& +x+(5.629719781 e-17) \\
y(x)= & -(0.003384338243) x^{8}-(0.001420180534) x^{7} \\
& +(0.06727516657) x^{6}-(0.1256152219) x^{5} \\
& -(0.2693617976) x^{4}+(2.000085149) x^{3} \\
& -(6.513886652 e-16) x^{2} \\
& +x-(1.153157431 e-15) .
\end{aligned}
$$

We compare the solutions found by the present method for $N=8, N=9$ and the absolute errors in Figure 1 and Table 1 . It is seen that when we increase integer $N$, the errors decrease.

Example 3 (see [30]). Now consider the linear Fredholm integro-differential-difference equation given by

$$
\begin{aligned}
y^{\prime \prime \prime}(x) & -(x-1) y^{\prime \prime}+(x-1) y^{\prime}(x)-y(x)+y^{\prime}(x-1) \\
= & e^{x-1}+x\left(e x-\frac{1}{e} x-2 \frac{1}{e}\right) \\
& +\int_{-1}^{1}\left(x t-x^{2}\right) y(t) d t \quad-1 \leq x, t \leq 1
\end{aligned}
$$


TABLE 1: Comparison of the absolute errors of Example 2.

\begin{tabular}{|c|c|c|c|c|c|}
\hline \multirow{3}{*}{$x_{i}$} & \multirow{3}{*}{ Exact solution } & \multicolumn{4}{|c|}{ Present method } \\
\hline & & \multicolumn{2}{|c|}{$N=8$} & \multicolumn{2}{|c|}{$N=9$} \\
\hline & & $y\left(x_{i}\right)$ & Absolute errors & $y\left(x_{i}\right)$ & Absolute errors \\
\hline-1 & -0.8414711 & -1.114125 & 0.2726539 & -3.078521 & 2.23705 \\
\hline-0.8 & -0.7173561 & -0.869866 & 0.1525099 & -1.875847 & 1.158491 \\
\hline-0.6 & -0.5646425 & -0.633677 & 0.06903453 & -1.054038 & 0.4893957 \\
\hline-0.4 & -0.3894183 & -0.4110374 & 0.0216191 & -0.5333391 & 0.1439208 \\
\hline-0.2 & -0.1986693 & -0.2014897 & 0.00282041 & -0.2163871 & 0.01771782 \\
\hline 0 & 0 & $5.62972 e-17$ & $5.62972 e-17$ & $-1.153157 e-15$ & $1.153157 e-15$ \\
\hline 0.2 & 0.1986693 & 0.2016525 & 0.00298318 & 0.2155338 & 0.01686445 \\
\hline 0.4 & 0.3894183 & 0.4137037 & 0.02428533 & 0.5200945 & 0.1306762 \\
\hline 0.6 & 0.5646425 & 0.6476974 & 0.08305494 & 0.9903835 & 0.4257410 \\
\hline 0.8 & 0.7173561 & 0.9164897 & 0.1991336 & 1.689322 & 0.9719655 \\
\hline 1 & 0.841471 & 1.235210 & 0.3937393 & 2.667579 & 1.826108 \\
\hline
\end{tabular}

TABLE 2: Comparison of the absolute errors of Example 5.3.

\begin{tabular}{|c|c|c|c|c|c|}
\hline \multirow{3}{*}{$x_{i}$} & \multirow{3}{*}{ Exact solution } & \multicolumn{4}{|c|}{ Present method } \\
\hline & & \multicolumn{2}{|c|}{$N=6$} & \multicolumn{2}{|c|}{$N=9$} \\
\hline & & $y\left(x_{i}\right)$ & Absolute errors & $y\left(x_{i}\right)$ & Absolute errors \\
\hline-1 & 0.3678794 & 0.368349 & $0.4695169 e-3$ & 0.3678795 & $9.388396 e-8$ \\
\hline-0.8 & 0.449329 & 0.4496545 & $0.3255685 e-3$ & 0.4493289 & $4.540547 e-8$ \\
\hline-0.6 & 0.5488116 & 0.5490096 & $0.1980008 e-3$ & 0.5488116 & $3.612436 e-8$ \\
\hline-0.4 & 0.67032 & 0.6704069 & $0.8684809 e-4$ & 0.67032 & $8.495309 e-9$ \\
\hline-0.2 & 0.8187308 & 0.8187468 & 0.1605797 & 0.8187308 & $7.845555 e-10$ \\
\hline 0 & 1.0 & 1.0 & 0 & 1.0 & 0 \\
\hline 0.2 & 1.221403 & 1.221369 & $0.3335666 e-4$ & 1.221403 & $3.813474 e-9$ \\
\hline 0.4 & 1.491825 & 1.491453 & $0.3721909 e-3$ & 1.491825 & $2.903571 e-8$ \\
\hline 0.6 & 1.822119 & 1.820404 & $0.171522 e-2$ & 1.822119 & $0.2499077 e-6$ \\
\hline 0.8 & 2.225541 & 2.220096 & $0.5445047 e-2$ & 2.225537 & $0.3627591 e-5$ \\
\hline 1 & 2.718282 & 2.704284 & 0.01399794 & 2.71826 & $0.2222775 e-4$ \\
\hline
\end{tabular}

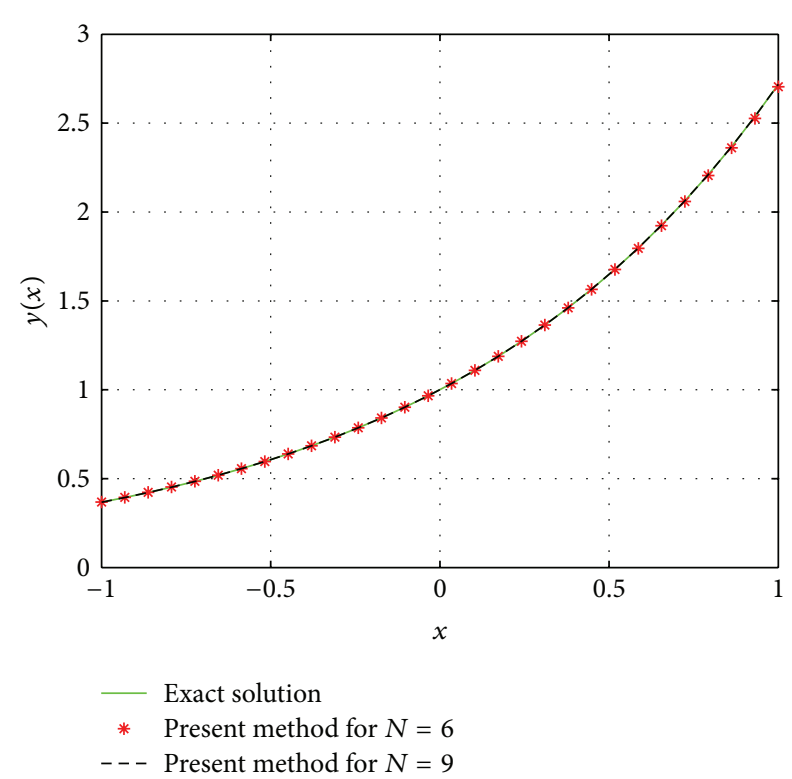

FIGURE 2: Numerical and exact solutions of Example 3 for $N=6,9$.

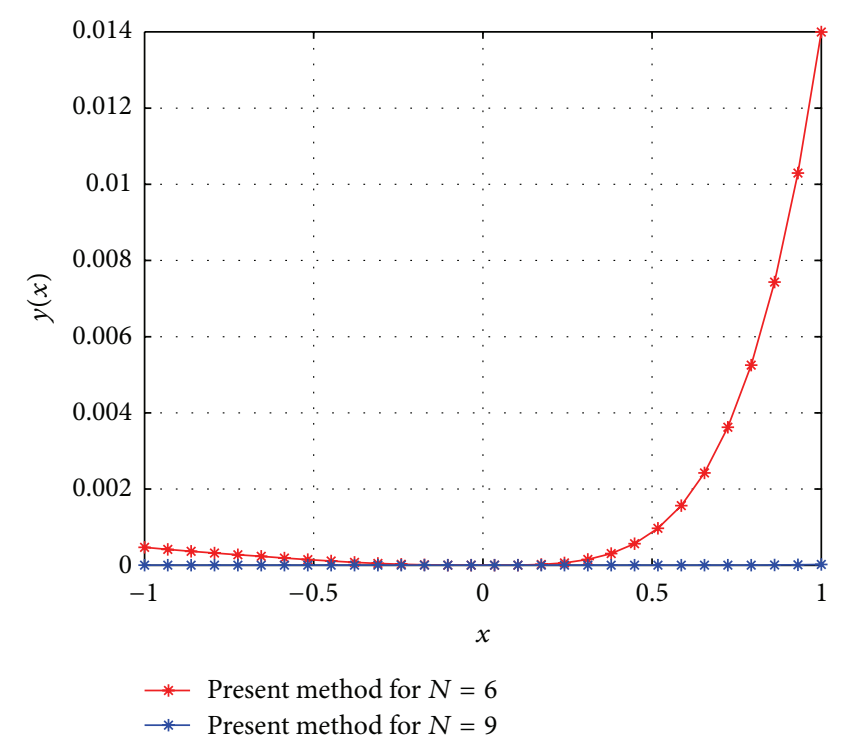

FIgURE 3: Comparison of the absolute errors of Example 3 for $N=$ 6,9 . 
with the initial conditions $y(0)=0, y^{\prime}(0)=1$, and $y^{\prime \prime}(0)=$ 0 and the exact solution $y(x)=e^{x}$.

Here

$$
\begin{gathered}
P_{0}(x)=-1, \\
P_{1}(x)=x-1, \\
P_{2}(x)=-(x-1), \quad P_{3}(x)=1, \\
Q_{1}(x)=1, \quad \mu_{1}=1, \tau_{1}=-1, \\
\lambda=1, \quad K(x, t)=x t-x^{2}, \\
g(x)=e^{x-1}+x\left(e x-\frac{1}{e} x-2 \frac{1}{e}\right)
\end{gathered}
$$

From (40), the fundamental matrix equation of the problem becomes

$$
\begin{aligned}
& \left\{\mathbf{P}_{0} \mathbf{X}\left(\mathbf{T}^{T}\right)^{0} \mathbf{C}^{T}+\mathbf{P}_{1} \mathbf{X}\left(\mathbf{T}^{T}\right)^{1} \mathbf{C}^{T}+\mathbf{P}_{2} \mathbf{X}\left(\mathbf{T}^{T}\right)^{2} \mathbf{C}^{T}\right. \\
& +\mathbf{P}_{3} \mathbf{X}\left(\mathbf{T}^{T}\right)^{3} \mathbf{C}^{T}+\mathbf{Q}_{1} \mathbf{X} \boldsymbol{\beta}(1,-1)\left(\mathbf{T}^{T}\right)^{1} \mathbf{C}^{T} \\
& \left.-\lambda \mathbf{X} \mathbf{C}^{T} \mathbf{K}_{f} \mathbf{Q}\right\} \mathbf{A}=\mathbf{G} .
\end{aligned}
$$

The solutions obtained for $N=6,9$ are compared with the exact solution is $e^{x}$ which are given in Figure 2 and we compare the absolute errors found by present method for $N=$ 6,9 in Figure 3. Also, the numerical solution and absolute errors are compared for $N=6,9$ in Table 2 .

\section{Conclusion}

The Fibonacci collocation method is used to solve the linear Fredholm integro-differential-difference equations numerically. The obtained numerical results show that the accuracy improves when $N$ is increased. Tables and figures indicate that as $N$ increases, the errors decrease more rapidly. A considerable advantage of the method is that the Fibonacci polynomial coefficients of the solution are found very easily by using computer programs. This method can also be extended to the system of linear integro-differentialdifference equation with variable coefficients, but some modifications are required.

\section{References}

[1] M. K. Kadalbajoo and K. K. Sharma, "Numerical analysis of boundary-value problems for singularly-perturbed differentialdifference equations with small shifts of mixed type," Journal of Optimization Theory and Applications, vol. 115, no. 1, pp. 145$163,2002$.

[2] C. E. Elmer and E. S. Van Vleck, "Traveling wave solutions for bistable differential-difference equations with periodic diffusion," SIAM Journal on Applied Mathematics, vol. 61, no. 5, pp. 1648-1679, 2001.

[3] D. D. Bainov, M. B. Dimitrova, and A. B. Dishliev, "Oscillation of the bounded solutions of impulsive differential-difference equations of second order," Applied Mathematics and Computation, vol. 114, no. 1, pp. 61-68, 2000.
[4] M. K. Kadalbajoo and K. K. Sharma, "Numerical analysis of singularly perturbed delay differential equations with layer behavior," Applied Mathematics and Computation, vol. 157, no. 1, pp. 11-28, 2004.

[5] M. T. Rashed, "Numerical solution of functional differential, integral and integro-differential equations," Applied Mathematics and Computation, vol. 156, no. 2, pp. 485-492, 2004.

[6] K. Maleknejad and Y. Mahmoudi, "Numerical solution of linear Fredholm integral equation by using hybrid Taylor and blockpulse functions," Applied Mathematics and Computation, vol. 149, no. 3, pp. 799-806, 2004.

[7] W. Wang and C. Lin, "A new algorithm for integral of trigonometric functions with mechanization," Applied Mathematics and Computation, vol. 164, no. 1, pp. 71-82, 2005.

[8] W. Wang, "An algorithm for solving the high-order nonlinear Volterra-Fredholm integro-differential equation with mechanization," Applied Mathematics and Computation, vol. 172, no. 1, pp. 1-23, 2006.

[9] C. E. Elmer and E. S. Van Vleck, “A variant of Newton's method for the computation of traveling waves of bistable differentialdifference equations," Journal of Dynamics and Differential Equations, vol. 14, no. 3, pp. 493-517, 2002.

[10] P. Rai and K. K. Sharma, "Numerical study of singularly perturbed differential-difference equation arising in the modeling of neuronal variability," Computers \& Mathematics with Applications, vol. 63, no. 1, pp. 118-132, 2012.

[11] P. Rai and K. K. Sharma, "Parameter uniform numerical method for singularly perturbed differential-difference equations with interior layers," International Journal of Computer Mathematics, vol. 88, no. 16, pp. 3416-3435, 2011.

[12] V. Kumar and K. K. Sharma, "An optimized B-spline method for solving singularly perturbed differential difference equations with delay as well as advance," Neural, Parallel \& Scientific Computations, vol. 16, no. 3, pp. 371-385, 2008.

[13] M. K. Kadalbajoo and K. K. Sharma, "An exponentially fitted finite difference scheme for solving boundary-value problems for singularly-perturbed differential-difference equations: small shifts of mixed type with layer behavior," Journal of Computational Analysis and Applications, vol. 8, no. 2, pp. 151-171, 2006.

[14] M. K. Kadalbajoo and K. K. Sharma, "Numerical analysis of boundary-value problems for singularly perturbed differentialdifference equations: small shifts of mixed type with rapid oscillations," Communications in Numerical Methods in Engineering, vol. 20, no. 3, pp. 167-182, 2004.

[15] M. H. Reihani and Z. Abadi, "Rationalized Haar functions method for solving Fredholm and Volterra integral equations," Journal of Computational and Applied Mathematics, vol. 200, no. 1, pp. 12-20, 2007.

[16] M. H. AliAbadi and E. L. Ortiz, "Numerical treatment of moving and free boundary value problems with the tau method," Computers \& Mathematics with Applications, vol. 35, no. 8, pp. 53-61, 1998.

[17] J. Pour-Mahmoud, M. Y. Rahimi-Ardabili, and S. Shahmorad, "Numerical solution of the system of Fredholm integrodifferential equations by the Tau method," Applied Mathematics and Computation, vol. 168, no. 1, pp. 465-478, 2005.

[18] S. M. Hosseini and S. Shahmorad, "Numerical solution of a class of integro-differential equations by the tau method with an error estimation," Applied Mathematics and Computation, vol. 136, no. 2-3, pp. 559-570, 2003. 
[19] K. Maleknejad and F. Mirzaee, "Numerical solution of integrodifferential equations by using rationalized Haar functions method," Kybernetes, vol. 35, no. 10, pp. 1735-1744, 2006.

[20] S. M. Hosseini and S. Shahmorad, "Tau numerical solution of Fredholm integro-differential equations with arbitrary polynomial bases," Applied Mathematical Modelling, vol. 27, no. 2, pp. 145-154, 2003.

[21] R. Farnoosh and M. Ebrahimi, "Monte Carlo method for solving Fredholm integral equations of the second kind," Applied Mathematics and Computation, vol. 195, no. 1, pp. 309-315, 2008.

[22] B. Asady, M. Tavassoli Kajani, A. Hadi Vencheh, and A. Heydari, "Solving second kind integral equations with hybrid Fourier and block-pulse functions," Applied Mathematics and Computation, vol. 160, no. 2, pp. 517-522, 2005.

[23] M. Sezer, "Taylor polynomial solutions of Volterra integral equations," International Journal of Mathematical Education in Science and Technology, vol. 25, no. 5, pp. 625-633, 1994.

[24] S. Yalçınbaş and M. Sezer, "The approximate solution of highorder linear Volterra-Fredholm integro-differential equations in terms of Taylor polynomials," Applied Mathematics and Computation, vol. 112, no. 2-3, pp. 291-308, 2000.

[25] M. Sezer and M. Gülsu, "Polynomial solution of the most general linear Fredholm integrodifferential-difference equations by means of Taylor matrix method," Complex Variables, vol. 50, no. 5, pp. 367-382, 2005.

[26] M. Gülsu and M. Sezer, "Approximations to the solution of linear Fredholm integrodifferential-difference equation of high order," Journal of the Franklin Institute, vol. 343, no. 7, pp. 720737, 2006.

[27] S. Yalçinbaş, "Taylor polynomial solutions of nonlinear Volterra-Fredholm integral equations," Applied Mathematics and Computation, vol. 127, no. 2-3, pp. 195-206, 2002.

[28] A. Akyüz-Daşcioğlu and M. Sezer, "Chebyshev polynomial solutions of systems of higher-order linear Fredholm-Volterra integro-differential equations," Journal of the Franklin Institute, vol. 342, no. 6, pp. 688-701, 2005.

[29] S. Yalçinbaş, M. Sezer, and H. H. Sorkun, "Legendre polynomial solutions of high-order linear Fredholm integro-differential equations," Applied Mathematics and Computation, vol. 210, no. 2, pp. 334-349, 2009.

[30] M. Gülsu and Y. Öztürk, "A new collocation method for solution of mixed linear integro-differential-difference equations," Applied Mathematics and Computation, vol. 216, no. 7, pp. 21832198, 2010.

[31] S. Yüzbaşı, N. Şahin, and M. Sezer, "Bessel collocation method for solving high-order linear Fredholm integro-differentialdifference equations," Journal of Advanced Research in Differential Equations, vol. 3, no. 2, pp. 1-23, 2011.

[32] K. Erdem, S. Yalçinbas, and M. Sezer, "A new collocation method for solution of linear Fredholm integro differentialdifference equations using Bernoulli polynomials," in Proceedings of the International Conference on Applied Analysis and Algebra (ICAAA), Yildiz Technical University, Istanbul, Turkey, 1992.

[33] A. Akyüz-Daşcioğlu and M. Sezer, "A Taylor polynomial approach for solving the most general linear Fredholm integrodifferential-difference equations," Mathematical Methods in the Applied Sciences, vol. 35, no. 7, pp. 839-844, 2012.

[34] S. Yalçınbaş, N. Özsoy, and M. Sezer, "Approximate solution of higher order linear differential equations by means of a new rational Chebyshev collocation method," Mathematical \& Computational Applications, vol. 15, no. 1, pp. 45-56, 2010.
[35] S. Yalçınbaş, M. Aynigül, and T. Akkaya, "Legendre series solutions of Fredholm integral equations," Mathematical \& Computational Applications, vol. 15, no. 3, pp. 371-381, 2010.

[36] A. Kurt, Fibonacci polynomial solutions of linear differential, integral and integro-differential equations [M.S. thesis], Graduate School of Natural and Applied Sciences, Mugla University, Mugla, Turkey, 2012. 


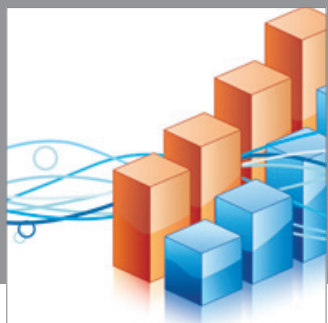

Advances in

Operations Research

mansans

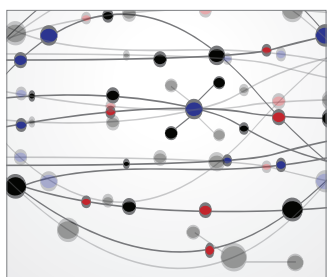

The Scientific World Journal
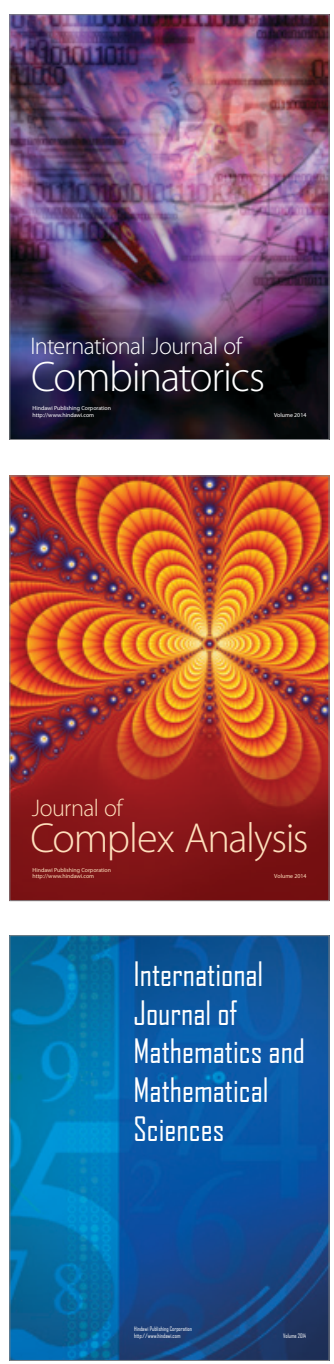
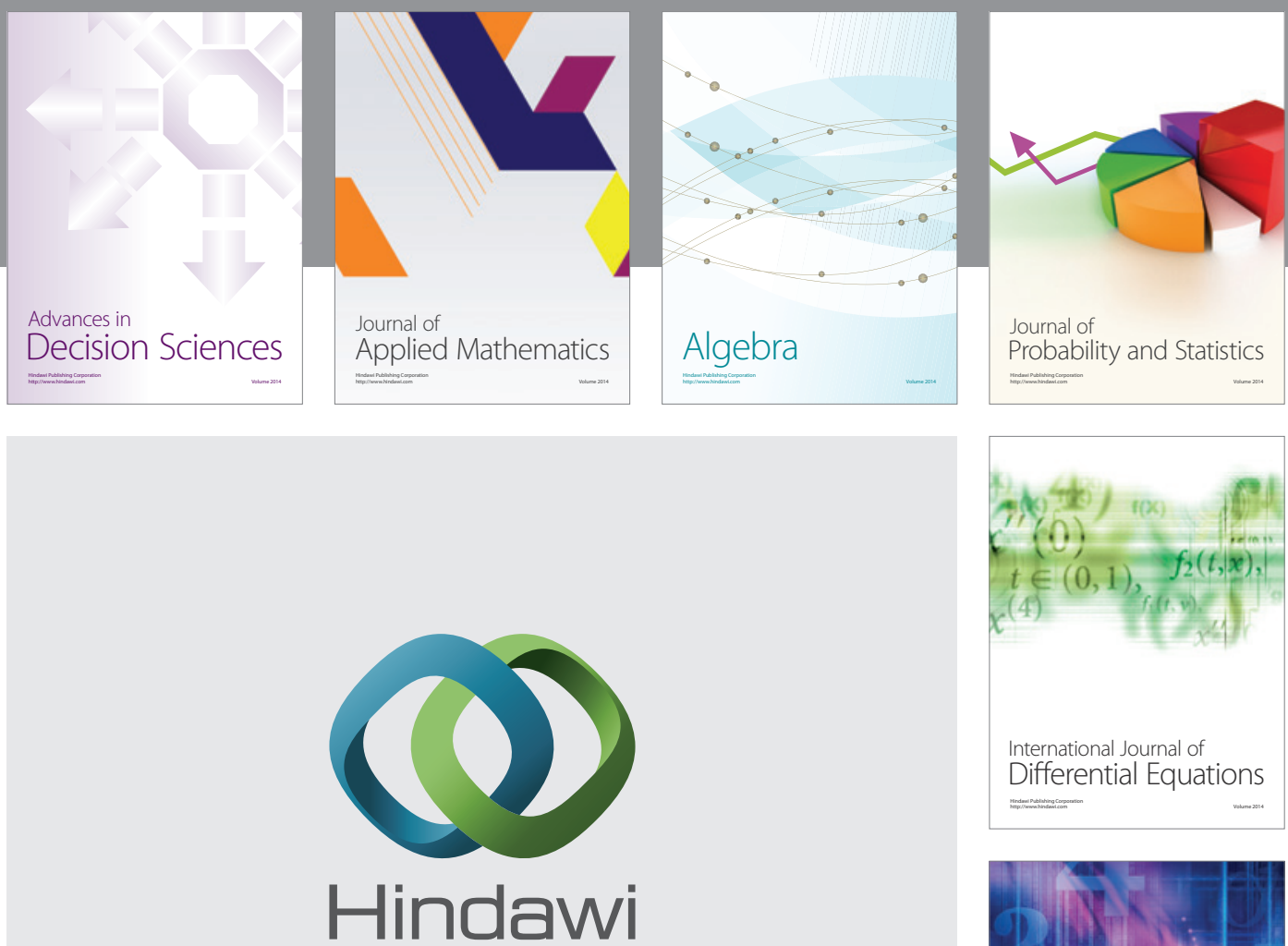

Submit your manuscripts at http://www.hindawi.com
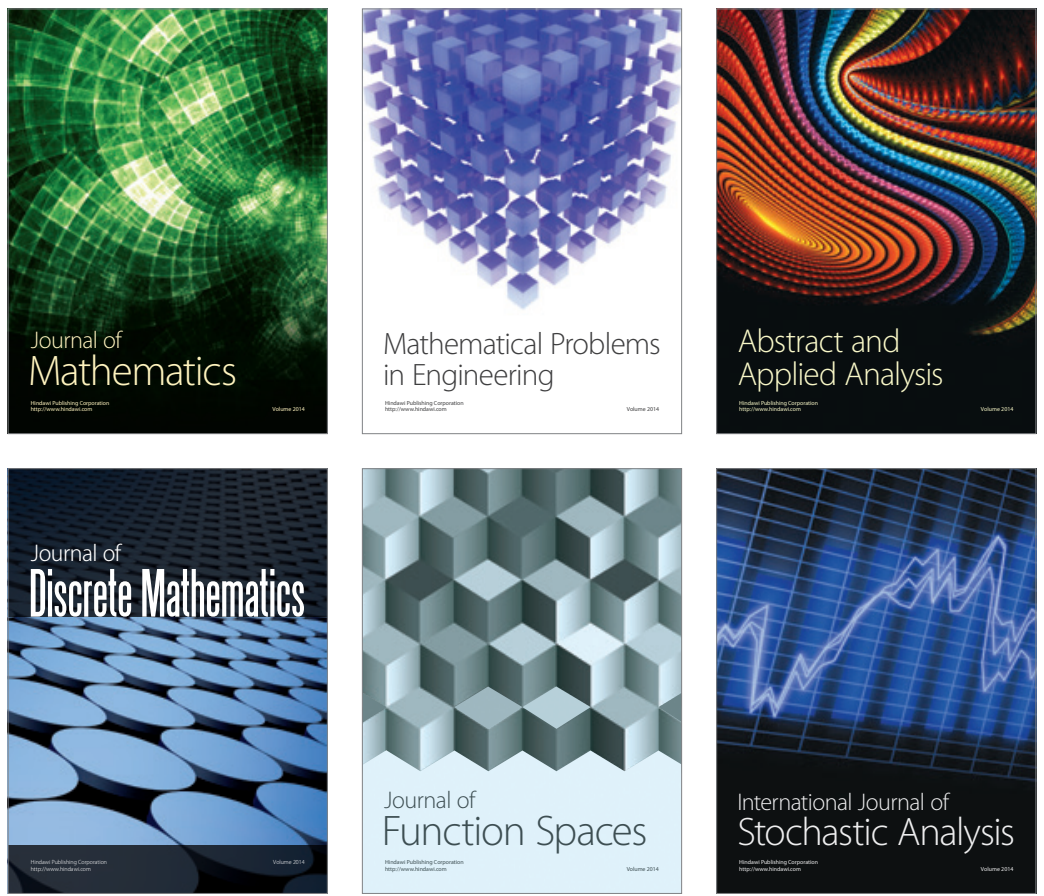

Journal of

Function Spaces

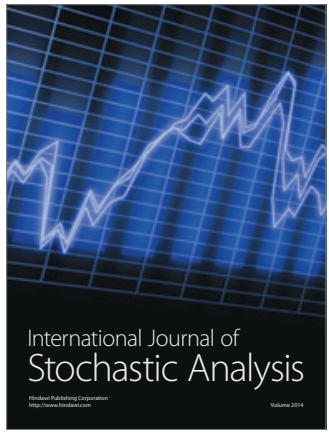

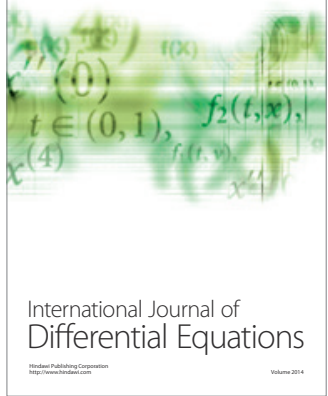
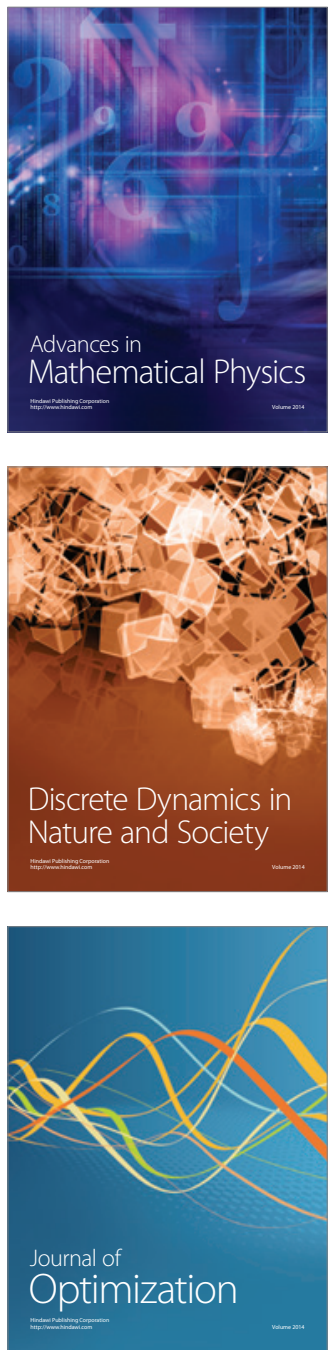